\title{
Role of light and heavy embedded nanoparticles on the thermal conductivity of SiGe alloys
}

\author{
A. Kundu, ${ }^{1}$ N. Mingo, ${ }^{1,2}$ D. A. Broido,${ }^{3}$ and D. A. Stewart ${ }^{4}$ \\ ${ }^{1}$ CEA-Grenoble, 17 Rue des Martyrs, Grenoble FR-38000, France \\ ${ }^{2}$ Department of Electrical Engineering, University of California, Santa Cruz, California 95064, USA \\ ${ }^{3}$ Department of Physics, Boston College, Chestnut Hill, Massachusetts 02467, USA \\ ${ }^{4}$ Cornell Nanoscale Facility, Cornell University, Ithaca, New York 14853, USA \\ (Received 8 June 2011; revised manuscript received 9 August 2011; published 9 September 2011)
}

\begin{abstract}
We have used an atomistic ab initio approach with no adjustable parameters to compute the lattice thermal conductivity of $\mathrm{Si}_{0.5} \mathrm{Ge}_{0.5}$ with a low concentration of embedded $\mathrm{Si}$ or Ge nanoparticles of diameters up to $4.4 \mathrm{~nm}$. Through exact Green's function calculation of the nanoparticle scattering rates, we find that embedding Ge nanoparticles in $\mathrm{Si}_{0.5} \mathrm{Ge}_{0.5}$ provides $20 \%$ lower thermal conductivities than embedding Si nanoparticles. This contrasts with the Born approximation, which predicts an equal amount of reduction for the two cases, irrespective of the sign of the mass difference. Despite these differences, we find that the Born approximation still performs remarkably well, and it permits investigation of larger nanoparticle sizes, up to $60 \mathrm{~nm}$ in diameter, not feasible with the exact approach.
\end{abstract}

DOI: 10.1103/PhysRevB.84.125426

PACS number(s): 66.70.Lm, 63.20.dk, 63.50.Gh

\section{INTRODUCTION}

Nanoparticle embedded in alloy thermoelectric (NEAT) materials have been proposed as a means of improving the thermoelectric properties of solid solutions. In a clear experimental demonstration of this concept, Kim et al. found a remarkable reduction of the thermal conductivity $(\kappa)$ of InGaAs upon the introduction of lattice-matched ErAs nanoparticles, below the alloy limit, without any decrease of the thermoelectric power factor. ${ }^{1}$ In some cases nanoparticles may also play an active role in increasing the power factor. ${ }^{2}$ Naturally forming nanoinclusions are also thought to be at the core of the rather high thermoelectric figure of merit $(Z T)$ of LAST (lead-antimony-silver-tellurium) materials. ${ }^{3}$ Theoretically, the introduction of nanophases inside alloys to reduce $\kappa$ and improve $Z T$ has been investigated using various approaches. ${ }^{4-7}$ However, these approaches approximate wave scattering via a continuum description, and they rely on adjustable parameters. Our aim is to overcome these drawbacks by performing a parameter-free atomistic calculation, which includes the nanoparticle scattering to all orders. We will show that the latter has a large influence on the results, which become highly asymmetric with respect to the sign of the scatterer's mass difference. (First-order perturbation theory yields a quadratic, symmetric dependence.) Thus the question of whether it is better to embed lighter or heavier nanoparticles becomes very relevant in the light of the full-order calculation. We provide the answer in the specific case of SiGe alloys, and we discuss its implications when developing novel NEAT materials.

Our parameter-free approach to compute thermal conductivity is based on an exact numerical solution of the linearized Boltzmann transport equation (BTE) for phonons. ${ }^{8-11}$ As a result of the computational challenge, many works still circumvent this solution by resorting to a host of approximations, most notably the relaxation-time approximation. ${ }^{12}$ A few years ago some of us showed that it is possible to predict the lattice thermal conductivity of group IV singlecrystal semiconductors from first principles, i.e., using the fundamental physical constants as the sole experimental inputs. ${ }^{9,10}$ In the present paper we extend our approach to the case of disordered solid solutions such as $\mathrm{Si}_{x} \mathrm{Ge}_{1-x}$, also including embedded nanoparticles. This requires the $a b$ initio calculation of elastic scattering rates due to both alloy disorder and the nanoparticles. Here we use atomistic Green's function techniques to compute those rates to all orders, beyond the Born approximation. We will show that the full result may deviate noticeably from the Born approximation.

\section{THEORY}

The thermal conductivity of a bulk material can be calculated as

$$
\kappa=\frac{1}{k_{B} T^{2}} \frac{V_{\mathrm{uc}}}{8 \pi^{3}} \sum_{\lambda} n_{0}\left(n_{0}+1\right)\left|v_{\lambda}^{z}\right|^{2} \hbar^{2} \omega_{\lambda}^{2} \tau_{\lambda},
$$

where $V_{\text {uc }}$ is the unit-cell volume, the summation sign is shorthand for $\sum_{\lambda^{\prime}} \equiv \sum_{\alpha^{\prime}} \int_{\mathrm{BZ}} d \vec{q}^{\prime}$ (the integral is performed over the volume of the Brillouin zone), and $\lambda$ stands for the phonon branch index and wave vector, $\{\alpha, \vec{q}\} . \omega_{\lambda}$ and $v_{\lambda}^{z}$ are, respectively, the frequency and the group velocity along the $z$ direction of the corresponding phonon and $n_{0}$ is their occupation number. The $\tau_{\lambda}$ are scattering times that contain all the information about the nonequilibrium phonon distribution. Details on the BTE and its solution have been given in Refs. $8-11$. We will just summarize it briefly. The equation to solve is

$$
\tau_{\lambda}=\tau_{\lambda}^{0}+\tau_{\lambda}^{0} \Delta_{\lambda}
$$

where

$$
\begin{aligned}
\Delta_{\lambda} \equiv & \sum_{\lambda^{\prime} \lambda^{\prime \prime}}^{+} \Gamma_{\lambda \lambda^{\prime} \lambda^{\prime \prime}}^{+}\left(\xi_{\lambda \lambda^{\prime \prime}} \tau_{\lambda^{\prime \prime}}-\xi_{\lambda \lambda^{\prime}} \tau_{\lambda^{\prime}}\right) \\
& +\sum_{\lambda^{\prime} \lambda^{\prime \prime}}^{-} \frac{1}{2} \Gamma_{\lambda \lambda^{\prime} \lambda^{\prime \prime}}^{-}\left(\xi_{\lambda \lambda^{\prime \prime}} \tau_{\lambda^{\prime \prime}}+\xi_{\lambda \lambda^{\prime}} \tau_{\lambda^{\prime}}\right)+\sum_{\lambda^{\prime}} \Gamma_{\lambda \lambda^{\prime}} \xi_{\lambda \lambda^{\prime}} \tau_{\lambda^{\prime}},
\end{aligned}
$$


where the $\Delta_{\lambda}$ term takes into account coupling of nonequilibrium $\lambda$ phonon modes to other phonon modes $\left(\lambda^{\prime}, \lambda^{\prime \prime}\right)$ based on energy and momentum conservation, and

$$
1 / \tau_{\lambda}^{0} \equiv \sum_{\lambda^{\prime} \lambda^{\prime \prime}}^{+} \Gamma_{\lambda \lambda^{\prime} \lambda^{\prime \prime}}^{+}+\sum_{\lambda^{\prime} \lambda^{\prime \prime}}^{-} \frac{1}{2} \Gamma_{\lambda \lambda^{\prime} \lambda^{\prime \prime}}^{-}+\sum_{\lambda^{\prime}} \Gamma_{\lambda \lambda^{\prime}}
$$

The (+) and (-) symbols over the sums in Eq. (4) indicate sums over $\lambda^{\prime}, \lambda^{\prime \prime}$ for the two types of three-phonon processes available, $\lambda \pm \lambda^{\prime} \leftrightarrow \lambda^{\prime \prime}$. The meanings of the three-phonon terms, $\Gamma_{\lambda \lambda^{\prime} \lambda^{\prime \prime}}^{+}$, and $\xi_{\lambda \lambda^{\prime \prime}}$ are given in Ref. 11 . The $\tau_{\lambda}$ were obtained through iterative solution of the BTE. For simplicity, the last term in $\Delta_{\lambda}$ [Eq. (3)] has been neglected. This term vanishes for the nearly isotropic elastic scattering of lowfrequency phonons, which dominate the thermal conductivity. The effect of elastic scattering from nanoparticles and alloy disorder is included in the sum of $\Gamma_{\lambda \lambda^{\prime}}$ in Eq. (4).

The main effect of a $\mathrm{Si}$ or Ge impurity or nanoparticle on lattice vibrations is through its mass difference compared to that of the host lattice. The dynamical equation for the displacements $u_{i}$ is $\omega^{2} \mathbf{M u}=\mathcal{K} \mathbf{u}$, where $\mathcal{K}_{i j}=\frac{\partial^{2} E}{\partial u_{i} \partial u_{j}}$ is the interatomic force constants matrix, and $M_{i j}=M_{i} \delta_{i j}$ is the mass diagonal matrix. When substitutional impurities of different mass are inserted, a diagonal matrix perturbation is added to the equation as $\mathcal{V}=-\left(\mathbf{M}^{\prime}-\mathbf{M}\right) \omega^{2}$, where $\mathbf{M}^{\prime}$ represents mass matrix of the impure system. This perturbation is nonzero only on the degrees of freedom associated with the scatterer. In the practical solution of the problem we work with the mass normalized matrices, $\mathbf{K} \equiv \mathbf{M}^{-1} \mathcal{K}, \mathbf{V} \equiv-\mathbf{M}^{-1} \mathcal{V}$. It has been shown that the other perturbation term, corresponding to the differences in force constants, $\mathcal{K}^{\prime}-\mathcal{K}$, has a much lesser effect on the thermal conductivity of SiGe alloys (about $10 \%$ of the total ${ }^{13}$ ). In the case of nanoparticles, an estimation of its order of magnitude can be easily made. Rayleigh scattering due to mass difference or to differences in the elastic constants have similar expressions, except for the prefactor, $S^{2}$. In the first case, this prefactor is $S_{\text {mass }} \sim\left(M_{n}-M_{m}\right) / M_{m}$. In the second case it is $S_{\mathrm{el}} \sim\left(\frac{v_{m}^{2}-\frac{M_{n}}{M_{m}} v_{n}^{2}}{v_{m}^{2}}\right)$, where subindices $m$ and $n$ stand for matrix and nanoparticle, respectively, and $v$ is the speed of sound. The presence of the $\frac{M_{n}}{M_{m}}$ term is needed to ensure that $S_{\mathrm{el}}$ is zero if only the atomic masses change, but not the interatomic force constants (IFCs). For $\mathrm{Si}_{0.5} \mathrm{Ge}_{0.5}$ we have $S_{\text {mass }}^{2}=0.4$, whereas $S_{L \text {,el }}^{2} \sim 0.01$ for longitudinal acoustic modes, and $S_{T \text {,el }}^{2} \sim 0.0004$ for transverse ones. Thus, the effect of different elastic constants is much smaller than that due to mass differences. Strain effects on the nanoparticle due to lattice mismatch could also induce additional scattering. In such cases, a factor of order $\sim \gamma\left(a_{n}-a_{m}\right) / a_{m}$ needs to be added to $S_{\mathrm{el}}$, where $\gamma \sim 1$ is the Grüneisen constant, and $a$ are the lattice constants of the nanoparticle and matrix materials, respectively. ${ }^{14}$ Addition of this term does not change the order of magnitude of $S_{\mathrm{el}}^{2}$, which remains smaller than $\sim 0.02$ and can be considered negligible compared with the mass difference effect. Taking into account the strain and IFC difference effects in an $a b$ initio calculation would require the self-consistent atomic relaxation of extremely large supercells, several times the size of the nanoparticles considered here. Therefore, given these difficulties and the minor resulting effect on the total thermal conductivity, the IFC differences will be neglected here.

The exact elastic scattering amplitudes due to a random distribution of independent scatterers in a homogeneous medium is $\Gamma_{\lambda \lambda^{\prime}} \equiv \sum_{p} f^{p} \Gamma_{\lambda \lambda^{\prime}}^{p}$ with

$$
\Gamma_{\lambda \lambda^{\prime}}^{p}=\frac{\Omega \pi}{2 \omega^{2}} \frac{1}{V_{p}}\left|\left\langle\lambda\left|\mathbf{T}_{\mathbf{p}}\left(\omega^{2}\right)\right| \lambda^{\prime}\right\rangle\right|^{2} \delta\left(\omega-\omega^{\prime}\right),
$$

where $f^{p}$ is the volume fraction of scatterers of type $p, V_{p}$ is the scatterer's volume, $\Omega$ is the volume into which the phonon eigenstates $|\lambda\rangle$ are normalized, and $\mathbf{T}_{\mathbf{p}}\left(\omega^{2}\right)$ is the $T$ matrix associated with the scatterer of type $p .{ }^{15}$ We have adopted a virtual crystal approximation (VCA) model for the medium, where the interatomic force constants and atomic masses of pure $\mathrm{Si}$ and $\mathrm{Ge}$ crystals are averaged according to their relative concentrations in the alloy. The total alloy scattering for bulk $\mathrm{Si}_{x} \mathrm{Ge}_{1-x}$ is given by the concentration weighted sum of the scattering probabilities of a Si impurity in the VCA medium, and a Ge impurity in the VCA medium: $\Gamma_{\lambda \lambda^{\prime}}^{\mathrm{SiGe}}=x \tilde{\Gamma}_{\lambda \lambda^{\prime}}^{\mathrm{Si}}+$ $(1-x) \tilde{\Gamma}_{\lambda \lambda^{\prime}}^{\mathrm{Ge}}$

The matrix $\mathbf{T}$ is defined in terms of the perturbation matrix $\mathbf{V}$ and the perturbed Green's function $\mathbf{G}^{+}$as $\mathbf{T}=$ $\mathbf{V}+\mathbf{V G}^{+} \mathbf{V}$, which, after some algebraic manipulations using the orthogonality and completeness of the eigenstates, can be expressed in terms of the unperturbed Green's function $\mathbf{g}^{+}\left(\omega^{2}\right)$ as

$$
\mathbf{T}\left(\omega^{2}\right)=\left[\mathbf{I}-\mathbf{V g}^{+}\left(\omega^{2}\right)\right]^{-1} \mathbf{V} .
$$

The integral form of the unperturbed Green's function $\mathbf{g}^{+}\left(\omega^{2}\right)$ is given by

$$
\mathbf{g}_{i j}^{+}\left(\omega^{2}\right)=\lim _{z \rightarrow \omega^{2}+i 0} \sum_{\lambda}\langle i \mid \lambda\rangle\langle\lambda \mid j\rangle z-\omega_{\lambda}^{2},
$$

where $|\lambda\rangle$ are the eigenstates of the infinite unperturbed lattice and $|i\rangle$ is a local displacement of the $i$ th degree of freedom in the direct lattice. For the numerical computation of $\mathbf{g}^{+}\left(\omega^{2}\right)$ in Eq. (7), we have employed the tetrahedron approach of Lambin and Vigneron. ${ }^{16}$ The total scattering rate due to the nanoparticles, appearing as the third term on the right-hand side of Eq. (4), is efficiently computed using the optical theorem: $:^{15}$

$$
1 / \tau_{\lambda}^{n p}=\sum_{\lambda^{\prime}} \Gamma_{\lambda \lambda^{\prime}}=\frac{\Omega}{2 \omega^{2}} \frac{f_{\mathrm{np}}}{V_{\mathrm{np}}} \operatorname{Im}\left[\left\langle\lambda\left|\mathbf{T}\left(\omega^{2}\right)\right| \lambda\right\rangle\right] .
$$

Most often in the literature, where the Born approximation is employed, the $T$ matrix is replaced by the perturbation matrix $V$. This is justified by the expansion $\mathbf{T} \simeq \mathbf{V}+\mathbf{V g V}+$ $\cdots$, valid for small perturbations. In this approximation one obtains

$$
\begin{aligned}
\frac{1}{\tau_{\lambda}^{\mathrm{np}}} & =\frac{\Omega}{16 \pi^{2}} g_{2} \omega_{\lambda}^{2} D_{\lambda}^{S}, \\
D_{\lambda}^{S} & =\sum_{\lambda^{\prime}}\left|\sum_{k^{\prime}} \mathbf{e}_{k^{\prime}}^{\lambda} \cdot \mathbf{e}_{k^{\prime}}^{\lambda^{\prime *}}\right|^{2}\left|S_{\Delta \mathbf{q}}\right|^{2} \delta\left(\omega_{\lambda^{\prime}}-\omega_{\lambda}\right) .
\end{aligned}
$$

Here, $g_{2}=f_{p}\left(1-M^{\prime} / M\right)^{2} ; D_{\lambda}^{S}$ is like a phonon density of states but weighted by the structure factor for the nanoparticle: $S_{\Delta \mathbf{q}}=\frac{1}{N_{p}^{2}} \sum_{l \in \mathrm{np}} e^{i \mathbf{R}_{l} \cdot \Delta \mathbf{q}}, N_{p}$ being the number of unit cells making up the nanoparticle. The $l^{\prime}$ sum is only over those 
unit cells of the virtual crystal occupied by the atoms of the nanoparticle and $\Delta \mathbf{q}=\mathbf{q}-\mathbf{q}^{\prime}$. The $\mathbf{e}_{k}^{\lambda}$ are phonon eigenvectors for the $k$ th atom in a unit cell. In the limit of a single atom impurity, Eq. (9) correctly reduces to the form derived previously by Tamura for the scattering rate of isotope impurities in cubic crystals. ${ }^{17}$

A consequence of the Born approximation is that the sign of the perturbation does not matter: a given percent of either increase or decrease of the scatterer's mass density with respect to the host's should produce the same result. This is not true when the exact $T$ matrix is employed, and large differences can occur with respect to the Born approximation for large mass difference, as our results show below.

\section{RESULTS AND DISCUSSION}

We have first computed the thermal conductivity of $\mathrm{Si}_{0.5} \mathrm{Ge}_{0.5}$ at 300 and $800 \mathrm{~K}$. The perturbative approach for isotopic impurities, ${ }^{10}$ where $g_{2} \sim 10^{-4}$, has for decades also been used for alloys, ${ }^{13}$ where one might question its validity since $g_{2}$ in the alloy is several orders of magnitude larger (for $\mathrm{Si}_{0.5} \mathrm{Ge}_{0.5}, g_{2}=0.2$ ). We have compared the $\mathrm{Si}_{0.5} \mathrm{Ge}_{0.5}$ scattering rates from Eq. (9) with those obtained using the full $T$-matrix method, and we find these to be close, as are the alloy thermal conductivities: $\kappa_{300 \mathrm{~K}}=10.62(T$ matrix $)$ vs 10.27 (Born) $\mathrm{W} / \mathrm{m} \mathrm{K}$ and $\kappa_{800 \mathrm{~K}}=6.07$ ( $T$ matrix) vs 6.0 (Born) $\mathrm{W} / \mathrm{m} \mathrm{K}$. Note that the alloy thermal conductivities are far lower than those of either bulk Si or bulk Ge because the alloy scattering is much stronger than the three-phonon scattering. As a result, $\Delta_{\lambda}$ in Eqs. (2) and (3) is small and $\tau_{\lambda} \approx \tau_{\lambda}^{0}$. These values are about $30 \%$ larger than the experimental values at the same concentration and temperatures $(\sim 7.5-8 \mathrm{~W} / \mathrm{m} \mathrm{K}$ at room temperature, $\sim 4.5-5 \mathrm{~W} / \mathrm{m} \mathrm{K}$ at $800 \mathrm{~K}) .^{5,13,18}$ There are several reasons for this. First, as already discussed at length in Sec. II, our neglect of differences in force constants may lead to somewhat higher values. Also, experimental samples contain a certain amount of impurities and defects which also lower the thermal conductivity. In addition, there is a considerable spread in experimental results from different sources, which further attests to the various unknown factors present in experimental measurements of alloy samples. Finally, a recent first-principles calculation of $\mathrm{SiGe}$ alloy thermal conductivity showed that the virtual crystal approach slightly underestimates phonon scattering in alloys. ${ }^{19}$

Our calculation shows that for single-atom scatterers, low-frequency phonons are well described by the Born approximation, with almost no difference between $\mathrm{Si}$ and $\mathrm{Ge}$ impurities. This is clearly seen in Fig. 1, for the case of the longitudinal acoustic branch. Although the scattering rate of Ge and Si single atoms differ importantly at high frequency, this does not lead to much difference in $\kappa$, since $\kappa$ is dominated by low-frequency phonons. It is only when we consider larger nanoparticles that differences become appreciable in the thermal conductivity. For nanoparticles of diameter 1.1 $\mathrm{nm}$, containing 38 atoms, scattering rates differ considerably between the $\mathrm{Si}$ and $\mathrm{Ge}$ cases, already above $5 \mathrm{THz}$ for $\omega$. This leads to a difference between the thermal conductivities of the corresponding composites. The difference becomes even more appreciable for larger nanoparticles (see Fig. 1). Figure 2 compares the Born approximation result, with the

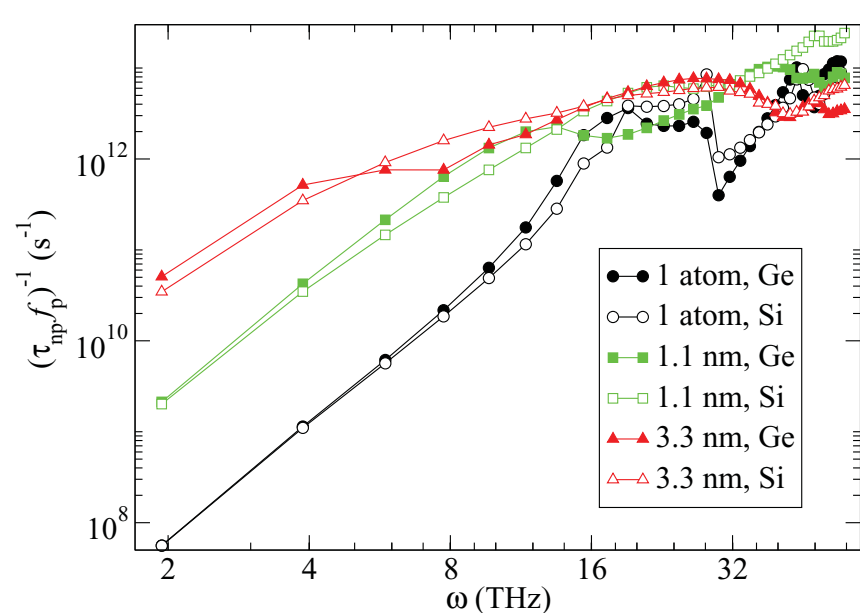

FIG. 1. (Color online) Comparison of the scattering rates $1 / \tau_{\lambda}^{\mathrm{np}}$ normalized by nanoparticle volume fraction $f_{p}$, due to $\mathrm{Ge}$ and $\mathrm{Si}$ nanoparticles of different sizes, in a $\mathrm{Si}_{0.5} \mathrm{Ge}_{0.5}$ matrix. The curves shown correspond to the LA phonon branch along direction (100).

exact scattering rates $1 / \tau_{\lambda}^{\mathrm{np}}$ for both a $\mathrm{Si}$ nanoparticle in $\mathrm{Si}_{0.5} \mathrm{Ge}_{0.5}$ and a $\mathrm{Ge}$ nanoparticle in $\mathrm{Si}_{0.5} \mathrm{Ge}_{0.5}$ for a diameter of $3.3 \mathrm{~nm}$, when the incident phonon direction is (100).

At high frequency both the $\mathrm{Si}$ and Ge cases deviate considerably from the Born approximation result (also shown in Fig. 2). This occurs when the wavelengths become comparable to the size of the scatterers, so we are no longer in the Rayleigh regime. An earlier interpolation formula had been proposed to link between the Born and geometric regime scattering cross sections: $6,20-23 \quad 1 / \sigma \simeq 1 / \sigma_{\text {geom }}+1 / \sigma_{\text {Born }}$. Figures 3 and 4 show $\kappa$ versus nanoparticle size obtained using this approximated interpolation formula. There is an optimal nanoparticle size that minimizes thermal conductivity at a given concentration. These $a b$ initio curves confirm the simpler model predictions in Ref. 7, yielding a minimum for a diameter of a few nanometers, and a slow increase after that.

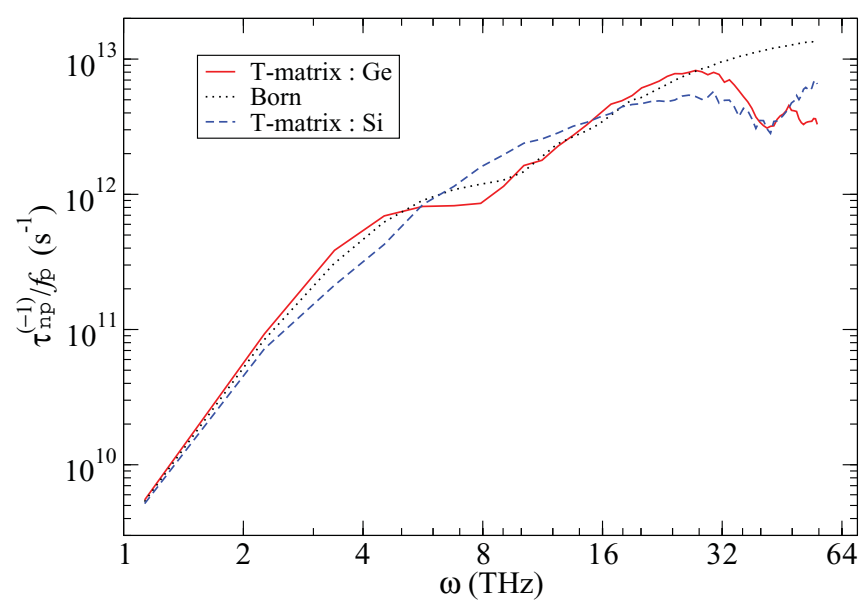

FIG. 2. (Color online) Scattering rate $1 / \tau_{\lambda}^{\mathrm{np}}$ due to $\mathrm{Ge}$ and $\mathrm{Si}$ nanoparticles of diameter $3.3 \mathrm{~nm}$, in a $\mathrm{Si}_{0.5} \mathrm{Ge}_{0.5}$ matrix, normalized by nanoparticle volume fraction $f_{p}$. The black dotted line is the Born approximation result from Eq. (9). The curves correspond to the LA phonon branch along direction (100). 


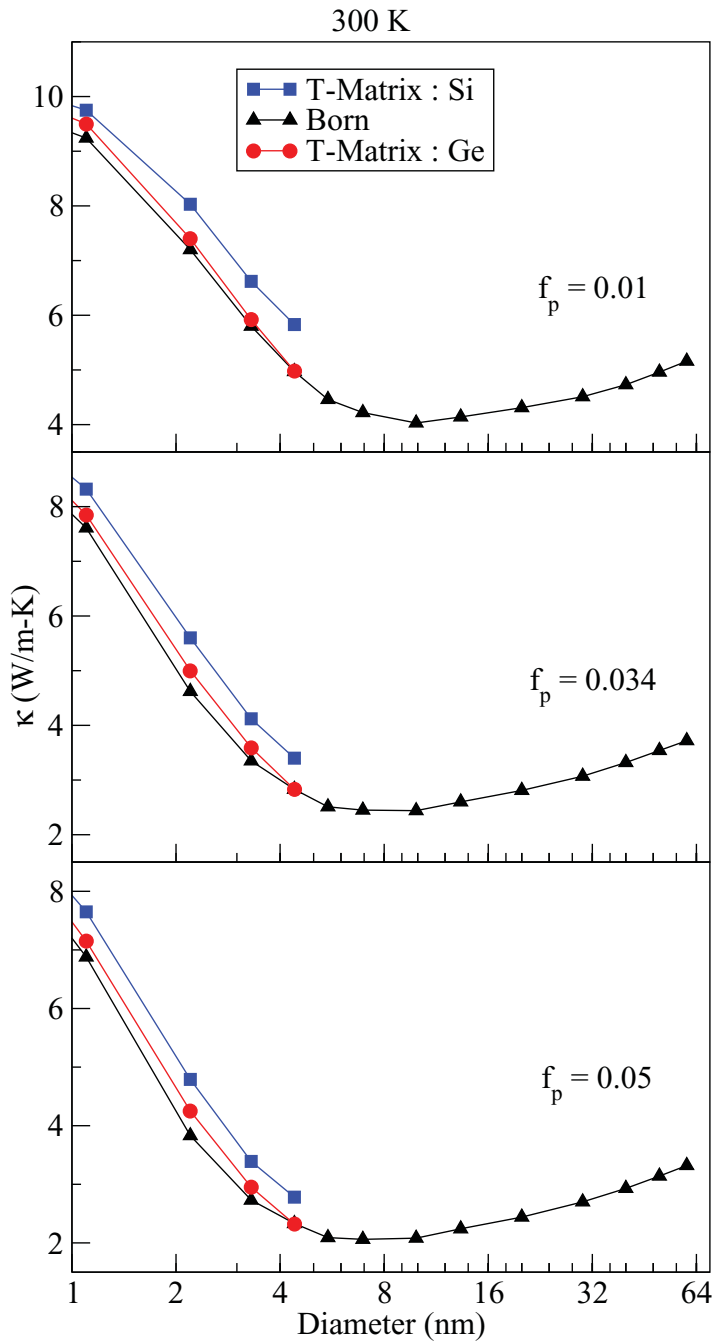

FIG. 3. (Color online) Thermal conductivity vs nanoparticle diameter at $1 \%$ (top), $3.4 \%$ (middle), and $5 \%$ (bottom) nanoparticle concentrations, for temperature $300 \mathrm{~K}$. Triangles: Born + geometrical interpolation. Squares: $T$-matrix calculation for $\mathrm{Si}$ nanoparticle. Circles: $T$-matrix calculation for Ge nanoparticle.

The interpolated expression still makes use of the Born approximation, so it does not inform us of possible differences between heavier and lighter scatterers. We have compared those results with the ones obtained using the $T$-matrix computed scattering rates. The plot shows quantitative differences, but the trends are the same. The $T$-matrix approach is very computationally demanding: a 4.4-nm-diameter particle, containing 2122 atoms is already at the limit of our computing capability. Therefore, we cannot assess the exact position of the minimum for the Ge or Si nanoparticle cases, although the graphs suggest that it may take place at a diameter between 5 and $10 \mathrm{~nm}$.

At equal nanoparticle size and concentration, the calculated $\kappa$ is always smaller for Ge (heavier) than for $\mathrm{Si}$ (lighter) nanoparticles. Their density difference with respect to $\mathrm{Si}_{0.5} \mathrm{Ge}_{0.5}$ is the same except for the sign, so in the framework of the Born approximation they should show an identical effect. This is clearly not the case, as shown in Fig. 5. This figure shows the ratio between the conductivities of the two

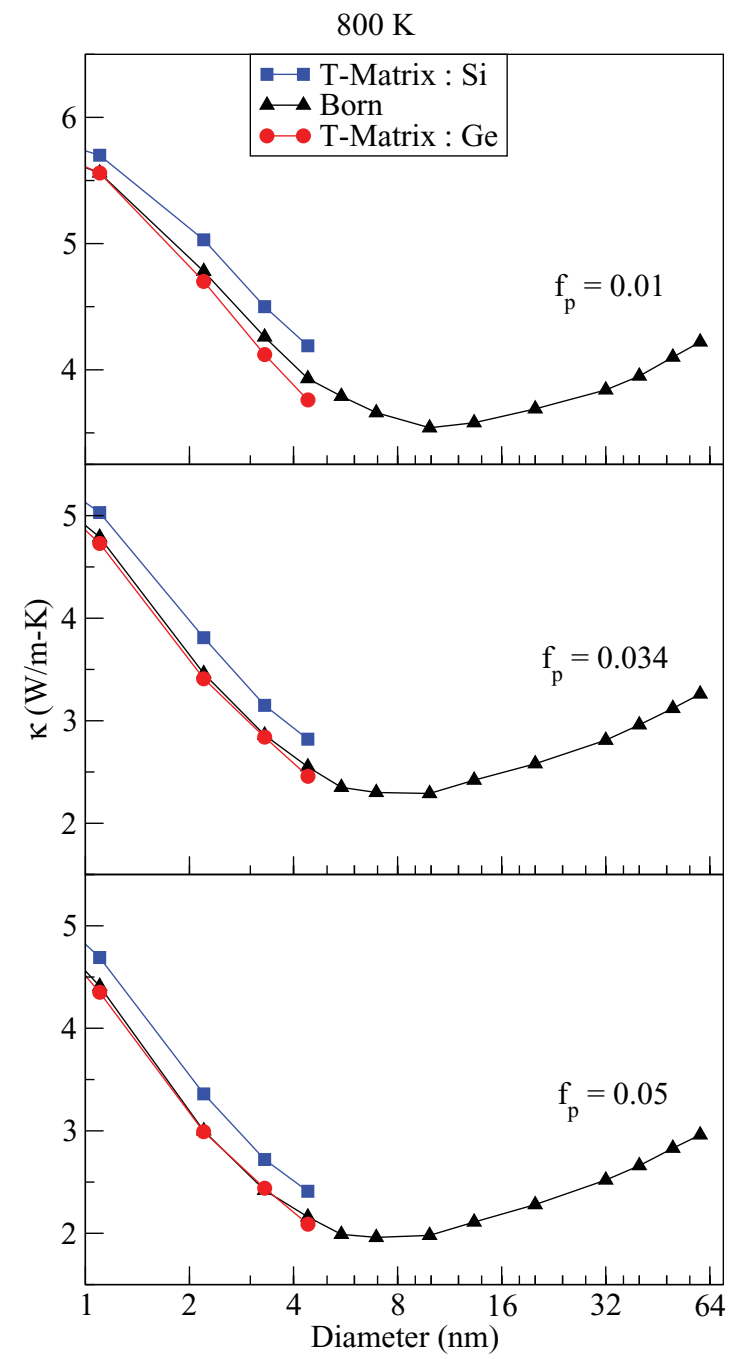

FIG. 4. (Color online) Thermal conductivity vs nanoparticle diameter at $1 \%$ (top), $3.4 \%$ (middle), and 5\% (bottom) nanoparticle concentrations, for temperature $800 \mathrm{~K}$. Triangles: Born + geometrical interpolation. Squares: $T$-matrix calculation for $\mathrm{Si}$ nanoparticle. Circles: $T$-matrix calculation for Ge nanoparticle.

cases, as a function of nanoparticle diameter: the thermal conductivity of the Ge nanoparticle case can be up to $20 \%$ lower than that of the Si nanoparticle case. The difference between the two cases highlights the very different densities of the particles and the medium, close to $20 \%$. The fact that $\mathrm{Ge}$ nanoparticles affect $\kappa$ more than Si nanoparticles is directly linked to their higher scattering rate at low frequency, visible in Fig. 2. The low frequencies are the ones that make the largest contribution to $\kappa$. This is because high frequencies already have very short mean free paths, and so most of the heat in the alloy is carried by low-frequency phonons. Thus, even though Si displays higher scattering rates at some intermediate and higher frequencies, it is the small scattering rates at low frequencies that determine the difference between the lighter and heavier types of nanocomposite.

A qualitative difference in the scattering rates of lighter and heavier impurities had been shown as early as 1963 for a model fcc scalar lattice with single and double impurities. ${ }^{24}$ Our results for the SiGe problem display the same kind of behavior, 


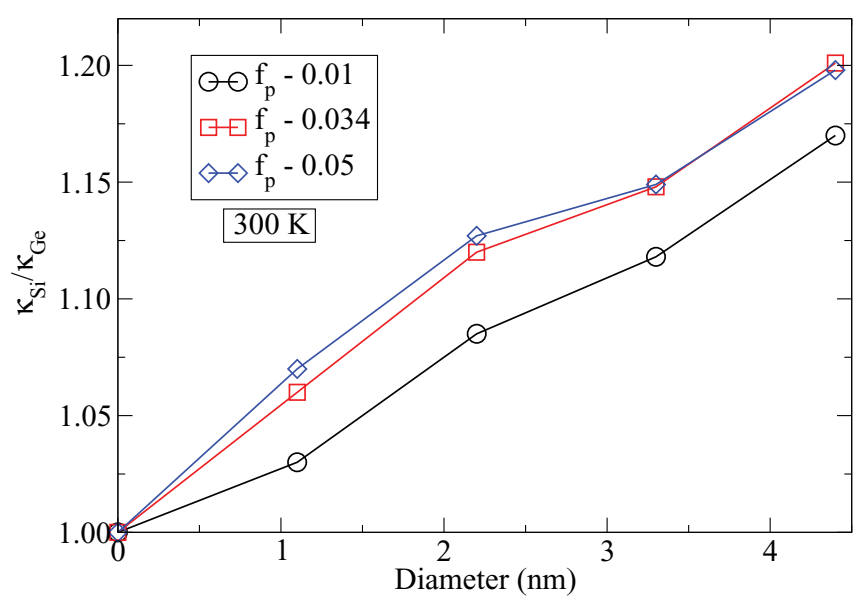

FIG. 5. (Color online) The ratio $\kappa_{\mathrm{Si}} / \kappa_{\mathrm{Ge}}$, of the thermal conductivity of a $\mathrm{SiGe}$ matrix with embedded $\mathrm{Si}$ nanoparticles, $\kappa_{\mathrm{Si}}$, to the thermal conductivity of a SiGe matrix with embedded Ge nanoparticles, $\kappa_{\mathrm{Ge}}$, is shown as a function of nanoparticle diameter, at $300 \mathrm{~K}$, for three different nanoparticle concentrations, $f_{p}$.

where the heavy impurities scatter more strongly than the light ones at low frequency. This can be qualitatively understood by making an analogy with electron scattering by a local potential. Heavier impurities are analogous to a potential well, whereas lighter ones are analogous to a potential barrier. From elementary scattering theory, the low-frequency scattering cross section of a potential well is larger than that of a potential hump, in agreement with the trend observed. Nevertheless, to our knowledge, the effect of arbitrarily sized nanoparticles on a realistic three-dimensional system has not previously been quantitatively investigated, and its consequences on thermal conductivity have not been addressed.

Some further comments are in order. In principle, the techniques presented here would also allow us to study other nanoparticle shapes and compositions. Our choice of pure
$\mathrm{Si}$ and Ge spherical nanoparticles has been motivated by simplicity. Experimentally, it may prove difficult to embed such nanoparticles into a SiGe matrix, because $\mathrm{Si}$ and Ge are fully miscible. Although high-concentration Ge nanoparticles with flat pyramidal or hemispherical shapes have been grown inside $\mathrm{Si}^{25}$ and $\mathrm{SiGe}$ matrix ${ }^{26}$ in the past, for the sake of clarity we have avoided introducing any experimentally determined morphological characteristics in our calculation.

\section{CONCLUSIONS}

We have preformed a parameter-free first-principles calculation of the thermal conductivity of SiGe alloys with embedded $\mathrm{Si}$ or Ge nanoparticles. In contrast with the commonly used Born approximation, it is found that embedding nanoparticles in the material affects its thermal conductivity differently depending on whether the nanoparticles are relatively heavier or lighter than the embedding matrix. The calculation predicts that heavier nanoparticles $(\mathrm{Ge})$ should be more efficient than lighter ones $(\mathrm{Si})$ in reducing the $\kappa$ of $\mathrm{Si}_{0.5} \mathrm{Ge}_{0.5}$. This behavior is determined by the higher scattering rate for heavier nanoparticles at low frequency, which is not predicted by the standard Born approximation, but is captured by the full Green's function calculation. Nevertheless, the approximated Born + geometrical approximation is found to work remarkably well, being within $20 \%$ of the exact result. The $a b$ initio calculation also confirms the existence of an optimal nanoparticle size that minimizes thermal conductivity, which had been previously predicted using a simpler model. ${ }^{7}$

\section{ACKNOWLEDGMENTS}

We acknowledge support from EU FP7 (NEAT, Grant No. 263440), Fondation Nanosciences, Agence Nationale de la Recherche, and the National Science Foundation. N.M. thanks Ali Shakouri for helpful discussions.
${ }^{1}$ W. Kim, J. Zide, A. Gossard, D. Klenov, S. Stemmer, A. Shakouri, and A. Majumdar, Phys. Rev. Lett. 96, 045901 (2006).

${ }^{2}$ M. Zebarjadi, K. Esfarjani, Z. Bian, and A. Shakouri, Nano Lett. 11, 225 (2011).

${ }^{3}$ K. F. Hsu, S. Loo, F. Guo, W. Chen, J. S. Dyck, C. Uher, T. Hogan, E. K. Polychroniadis, and M. G. Kanatzidis, Science 303, 818 (2004).

${ }^{4}$ L. A. Turk and P. G. Klemens, Phys. Rev. B 9, 4422 (1974).

${ }^{5}$ G. A. Slack and M. A. Hussain, J. Appl. Phys. 70, 2694 (1991).

${ }^{6}$ W. Kim and A. Majumdar, J. Appl. Phys. 99, 084306 (2006).

${ }^{7}$ N. Mingo, D. Hauser, N. P. Kobayashi, M. Plissonnier, and A. Shakouri, Nano Lett. 9, 711 (2009).

${ }^{8}$ M. Omini and A. Sparavigna, Nuovo Cimento D 19, 1537 (1997).

${ }^{9}$ D. A. Broido, M. Malorny, G. Birner, N. Mingo, and D. A. Stewart, Appl. Phys. Lett. 91, 231922 (2007).

${ }^{10}$ A. Ward, D. A. Broido, D. A. Stewart, and G. Deinzer, Phys. Rev. B 80, 125203 (2009).

${ }^{11}$ L. Lindsay, D. A. Broido, and N. Mingo, Phys. Rev. B 82, 161402(R) (2010).

${ }^{12}$ J. M. Ziman, Electrons and Phonons (Clarendon, London, 1962).
${ }^{13}$ B. Abeles, Phy. Rev. 131, 1906 (1963).

${ }^{14}$ P. G. Klemens, Proc. Phys. Soc. A 68, 1113 (1955).

${ }^{15}$ N. Mingo, K. Esfarjani, D. A. Broido, and D. A. Stewart, Phys. Rev. B 81, 045408 (2010).

${ }^{16} \mathrm{Ph}$. Lambin and J. P. Vigneron, Phys. Rev. B 29, 3430 (1984).

${ }^{17}$ S. I. Tamura, Phys. Rev. B 27, 858 (1983).

${ }^{18}$ I. Yonenaga, T. Akashi, and T. Goto, J. Phys. Chem. Solids 62, 1313 (2001).

${ }^{19}$ J. Garg, N. Bonini, B. Kozinsky, and N. Marzari, Phys. Rev. Lett. 106, 045901 (2011).

${ }^{20}$ J. W. Schwartz and C. T. Walker, Phys. Rev. 155, 969 (1967).

${ }^{21}$ J. W. Vandersande, Phys. Rev. B 15, 2355 (1977).

${ }^{22}$ Y. P. Joshi, Phys. Status Solidi B 95, 627 (1979).

${ }^{23}$ A. Majumdar, ASME Trans. J. Heat Transfer 115, 7 (1993).

${ }^{24}$ S. Takeno, Prog. Theor. Phys. 29, 191 (1963).

${ }^{25}$ G. Pernot, M. Stoffel, I. Savic, F. Pezzoli, P. Chen, G. Savelli, A. Jacquot, J. Schumann, U. Denker, I. Mönch, Ch. Deneke, O. G. Schmidt, J. M. Rampnoux, S. Wang, M. Plissonnier, A. Rastelli, S. Dilhaire, and N. Mingo, Nat. Mater. 9, 491 (2010).

${ }^{26}$ D. Hauser, M. Plissonnier, L. Montes, J. Simon, and G. Savelli (unpublished). 